\title{
Sudden hearing loss due to bee sting: A rare case
}

\author{
Erkan Yıldız ${ }^{1}$, Orhan Kemal Kahveci
}

\begin{abstract}
Sudden hearing loss (SHl); Define as, at least $30 \mathrm{~dB}$ sensorineural hearing loss in 3 consecutive frequencies. In general, idiopathic, viral and vascular causes are at the forefront. Local or systemic hypersensitivity reactions due to insect bites such as bee sting may occur. Sudden hearing loss is a more unknown finding in the literature due to bee sting. The first choice in treatment is systemic or local corticosteroid uses.
\end{abstract}

In this case report, the patient who had bilateral sudden hearing loss following bee sting and treatment approaches have been presented.

Keywords: Bee sting, sudden hearing loss, steroids

\section{Introduction}

Sudden hearing loss (SHL) or sudden sensorineural hearing loss (SHL) defined as; Hearing thresholds in 3 days consecutively in 3 frequencies more than $30 \mathrm{~dB}$ loss of hearing level. The incidence of SHL was reported avarage 5-20 / 100,000 in the literature. The average age for SHL is close to 50-60 ages between men and women. Due to etiology, viral infections, vascular occlusion, autoimmune diseases, metabolic diseases, neoplastic, toxic and neurological diseases may play role in SHL (1). In the diagnosis, high level noise exposure, history of infection, trauma and systemic diseases should be questioned. In this case, diagnosis is made by the audiometric test by showing the hearing loss. Autoacoustic emission and Bera tests also be used in patients who cannot perform audiometry. A significant number of patients have complaints of tinnitus. Blood tests performed in most patients have no effect on etiology and treatment. The only imaging that can help with the diagnosis is MRI and should be asked to all patients. Cerebellopontin corner tumors and other neoplastic changes, vascular-ischemic causes, labyrinthitis due to infection can be seen very clearly in MRI. Vestibular tests can be performed for patients with dizziness (2). Sudden hearing loss is an otologic emergency situation. The treatment should be started as soon as possible. The most important solution for the treatment is systemic corticosteroids. Because, they are idiopathic and do not require the treatment for the cause. Intratympanic steroids can also be given to those with systemic disease. Alcohol, smoking should be prohibited. In addition, vitamins, antiviral treatments, hyperbaric oxygen therapy, vasodilators and autoimmune treatments may planned $(1,2,3)$.
In patients with sudden hearing loss, the $32-65 \%$ of patients may improve without any treatment. The presence of vertigo, involvement of low frequencies, advanced hearing loss and increased sedimentation are the criteria of poor prognosis $(4,5)$. Reactions related to bee stings may occur depending on Ig E dependent or independent. Localized reactions can also lead to fatal general reactions such as anaphylactic shock.Gullian-Barre-like myelitis, hemolytic anemia, interstitial nephritis, encephalomyelitis and peripheral neuropathy have been detected in the literature for some patients. Earlier hearing loss due to bee sting was reported in one case. Venom immunotherapy and systemic steroids, as well as antihistamines, can be used to reduce the severity of symptoms in the treatment of bee stings.

\section{Case}

A 38-year-old female was admitted to the emergency department, one day after stinging of bees from the left auricle. Intramuscular phenyiramine maleate $45.5 \mathrm{mg} / 2 \mathrm{ml}$ + Dexamethasone $2 \mathrm{ml} / \mathrm{Mg} 1 * 1$ was administered in the emergency department. In the otoscopic examination, bilateral tympanic membrane was intact. Hemogram and biochemistry tests were normal. Bilateral total sensorineural hearing loss was present in the pure voice audiometry. The oacoustic emission test was negative in both ears. In bilateral tympanogram, while bilateral Type A tympanogram was obtained, reflexes could not be bilateral. Cranial and acoustic MRI have been reported as normal. The $1 \mathrm{mg} / \mathrm{kg}$ prednisolone treatment and proton pump inhibitor (esomeprezol $1 * 1$ p.o) were started. Audiometry and otoacoustic emissions of patient were normal. 


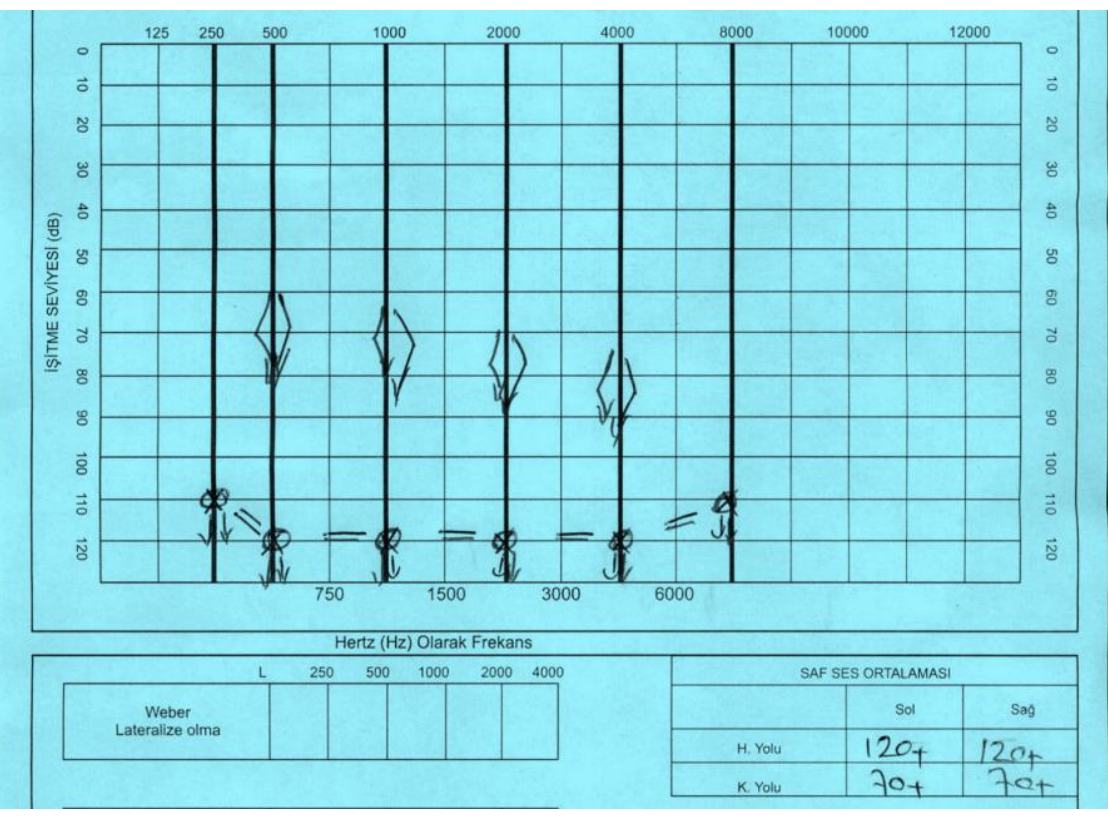

Figure 1: Audiometry measurement of Patient after bee sting

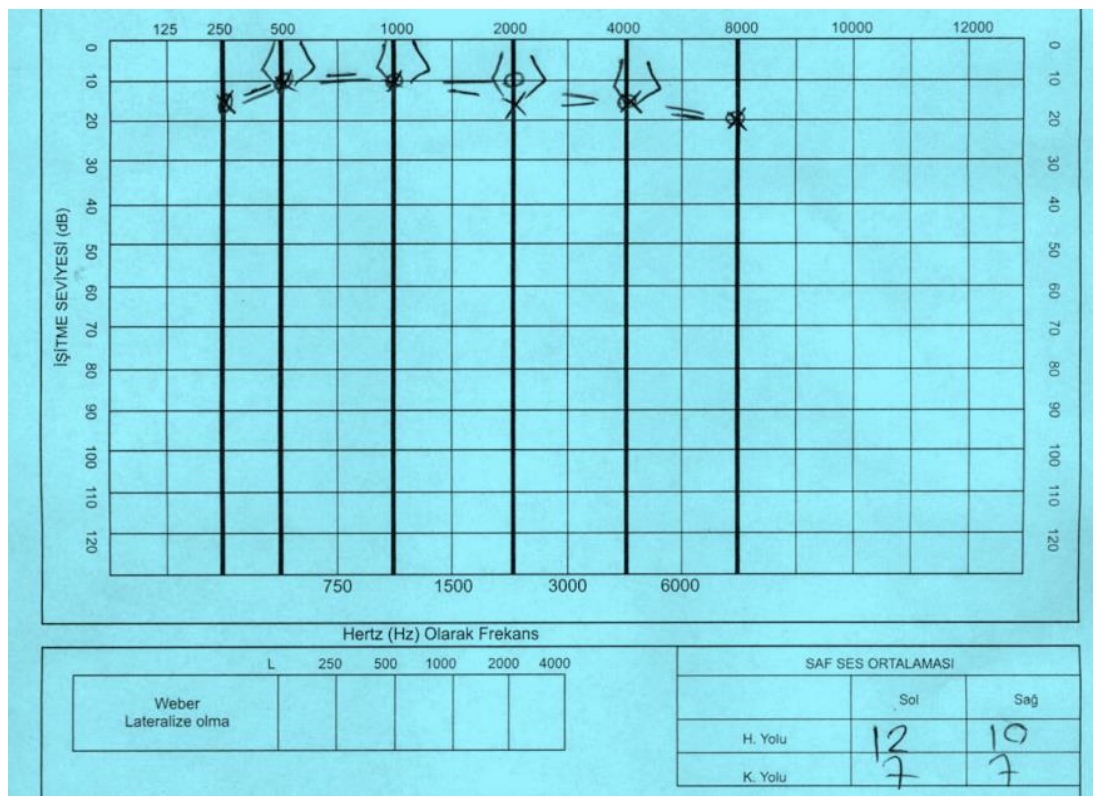

Figure 2: Audiometry measurement of patient was performed after 1 week of treatment

\section{Discussion}

After the bee sting, toxic, immunological and allergic reactions, anaphylaxis and shock may change the patient situation. Hypertension, myocardial infarction and multiorgan insufficiency may develop after Type 1 anaphylactic shock caused by bee sting from head and neck. Although insect bites have common effects, serious effects are rare. Optic neuritis, convulsion, lesions similar to Henoch Schonlein purpura, neuropathies similar to Guillain Barre syndrome, intravascular coagulation, compartment syndrome have been reported in the literature. But so far, no toxic effects have been reported on the cochlea. We could not detect any cause of sudden hearing loss because of bee sting in the literature.
Sudden sensorineural hearing loss is defined as sensorineural hearing loss of at least $30 \mathrm{~dB}$ at 3 consecutive frequencies occurring within 3 days $(1,2)$. It is usually accompanied by tinnitus and vertigo $(3,4)$. Sudden hearing loss, which usually occurs unilaterally, is usually seen in $65 \%$ of patients and symptoms return back with or without treatment. Losses at low frequency have a better prognosis than at high frequencies $(4,5)$. In our patient, there was total hearing loss affecting all frequencies in both ears. etiopathogenetic factors such as autoimmune diseases, infectious diseases, trauma, circulatory microvascular disorders, ototoxicity, neoplasms, transient obstructive membrane rupture and viral infections can be cause of SHL. The other $90 \%$ is called idiopathic sudden sensorineural hearing loss $(1,2,3)$. 
Apart from viral and vascular causes, etiology is the main cause of etiology. (4) None of the reasons described in our patient were present, only one case was reported in the literature (7).

Cause of sudden hearing loss cannot be found for most patients. Therefore, the treatment should be planned comprehensively such that it responds to all causes. In sudden hearing loss, vasodilators, volume expanders, antivirals and anticoagulants, together with steroids, constitute the most important part of the treatment $(4,5)$. Treatment approaches and molecular mechanism is still not fully clear. Due to literature, the full recovery rate of SHL is $25 \%$, the probability of partial recovery is $25 \%$ and the probability of not recovering is $50 \%$.

The fact that our patient responded faster than the other sudden hearing loss cases suggested that the steroid was related to both the sudden hearing loss treatment and its use in general for anaphylaxis. In our patient, a complete improvement in the audiometry was achieved. Also, oral prednisolone therapy may use in the treatment of hearing loss due to bee sting.

\section{Conclusion}

Systemic examination of patients with bee sting, as well as systematic otolaryngological examination is inevitable treatment plan for the benefit of patient. For better response for SHL treatment due to insect stings, treatment should be immediately started. Regression of symptoms depends on the time of initiation of treatment.

\section{Consent}

All images are entirely anonymized and the individual cannot be identified. No personal information of the individual was included.

Ethical statement: All procedures performed in studies involving human participants were in accordance with the ethical standards of the institutional and/ or national research committee and with the 1964 Helsinki declaration and its later amendments or comparable ethical standards. Funding statement The authors received no funding for this work.
Conflict of Interest: The authors declare no potential conflicts of interest with respect to the research, authorship, and/or publication of this article.

Author's Contributions: EY, OKK; Research concept and design, Patient examination, Research the literature, preparation of the article. EY; Revision of the article.

\section{References}

1. Fetterman BL, Saunders JE, Luxford WM. Prognosis and treatment of sudden sensorineural hearing loss. The American journal of otology. 1996 Jul;17(4):529-36.

2. Schuknecht HF, Donovan ED. The pathology of idiopathic sudden sensorineural hearing loss. Archives of oto-rhino-laryngology. 1986 Mar 1;243(1):1-5.

3. Ruckenstein MJ. Autoimmune inner ear disease. Current opinion in otolaryngology \& head and neck surgery. 2004 Oct 1;12(5):426-30.

4. Stachler RJ, Chandrasekhar SS, Archer SM, Rosenfeld RM, Schwartz SR, Barrs DM, Brown SR, Fife TD, Ford P, Ganiats TG, Hollingsworth DB. Clinical practice guideline: sudden hearing loss. Otolaryngology-Head and Neck Surgery. 2012 Mar;146(3_suppl):S1-35.

5. Nosrati-Zarenoe R, Hultcrantz E. Corticosteroid treatment of idiopathic sudden sensorineural hearing loss: randomized triple-blind placebo-controlled trial. Otology \& Neurotology. 2012 Jun $1 ; 33(4): 523-31$

6. Gezer D, Şenel E, Süslü İ. Arı sokmalarına karşı oluşan reaksiyonlar. Genel Tip Dergisi. 2012 Sep 1;22(3).

7. Düzenli U, Bozan N, Ayral A, Yalınkılıç A, Kıroğlu AF. A honey bee can threat ear: Sudden sensorineural hearing loss. The American journal of emergency medicine. 2017 Nov 1;35(11):1788-e1. 